\title{
The Application and Perspective of Multimedia Technology in Chemistry Experimental Instruction in China
}

\author{
Dimei Chen ${ }^{1}$, Xia Chen ${ }^{2}$, Wenxia Gao ${ }^{1}$ \\ ${ }^{1}$ College of Chemistry and Material Engineering, Wenzhou University, Wenzhou, China \\ ${ }^{2}$ Yuhuan Middle School, Taizhou, China \\ Email: cdm@wzu.edu.cn, bigxia1314@126.com
}

Received January $26^{\text {th }}, 2013$; revised February $28^{\text {th }}, 2013$; accepted March $14^{\text {th }}, 2013$

\begin{abstract}
Copyright (C) 2013 Dimei Chen et al. This is an open access article distributed under the Creative Commons Attribution License, which permits unrestricted use, distribution, and reproduction in any medium, provided the original work is properly cited.
\end{abstract}

\begin{abstract}
The paper elaborates the features and superiority of applying multimedia technology to chemistry experiment instruction in details, and introduces the practical application of multimedia technology in demonstration experiment. The application of multimedia technology in chemistry experimental instruction has its own features, namely enjoyment, great information capacity, sharing network, easy operation and flexibility. The paper concludes the superiority of applying multimedia technology to the chemistry experiment instruction from nine perspectives. Meanwhile, the paper elaborates its disadvantages. Simulated experiments cannot take place of real experiments. The relationship among teachers, students and multimedia needs to be dealt with properly, and the multimedia courseware should be scientific. Prospective forecast of multimedia technology in chemistry experimental instruction is also demonstrated.
\end{abstract}

Keywords: Multimedia Technology; Chemistry Experiment; Instrument; Simulation

\section{Introduction}

Multimedia technology used in traditional chemistry teaching was the integration of modern information technology and chemistry instruction. Meanwhile, it is an essential symbol of achieving educational informatization, and is an important approach to improve chemistry experimental teaching. Application of multimedia technology to traditional chemistry experiment has the advantage of establishing valid teaching scenario and stirring up the interest, which leads students' thought and improves the development of students' scientific literacy. There is no doubt that the integration of multimedia technology and teaching is a new trend and is a very effective way to develop students' self-study ability. However, we cannot simply apply multimedia technology to teach chemistry experiment. The information technology era promoted the development of education. The application of multimedia technology started in early 1980s in China and great progress has been made.

\section{Characteristics of the Application of Multimedia Technology in Chemistry Experimental Instruction}

Multimedia technology provides a good personal learning environment. Softwares are developed with the interactive digital technology to be used in both instruction and self-study (Huang, 2007). We can simulate experimental phenomena and wrong operations to improve students' understanding of experimental phenomena and results and cultivate their intellectual capacity and passion for experiment in the game-like environment (Ma \& Ran, 2008). The application of multimedia technology in chemistry experimental instruction has its own characteristics:

\section{Enjoyment (Yan, Yu, \& Sun, 2005)}

Multimedia technology can stimulate students' interest in chemistry by showing animation, pictures and sound. So the atmosphere of the classroom is activated to help students gain knowledge naturally.

\section{Great Information Capacity}

Using multimedia technology in teaching can save a lot of time in blackboard-writing. This kind of teaching model can impart amount of chemistry knowledge to students in regular class time. It also has good effect upon students' acceptance of new knowledge.

\section{Network Sharing}

With the development of Internet technology and information technology, it's universal for teachers and students to share resources on the internet. If the computer network and teaching software can be used reasonably, excellent teaching production can be shared by teachers and students all over the world (He, 2007), the long-distance education and autonomous learning will also come true (Wang, 2008). Therefore, students can learn online at any time, and they don't have to be limited by the forty-five minutes in classes.

\section{Easy Manipulation}

With the teacher's development of skills in the use of computers, the ability of using multimedia courseware also gets 
improved day by day. Technologies such as flash animation, digital pictures and digital cameras have been applied widely in multimedia courseware.

\section{Flexibility}

Multimedia courseware relates to theoretical knowledge with practice by taking video and photos and so on. To some degree, it provides an effective way for students to master professional knowledge and skills.

\section{The Situation of the Application of Multimedia Technology in Chemistry Experimental Instruction}

\section{Enhancing Visibility and Improving Experiment Results}

As we all know, some reaction speed of experiments is too fast to observe the phenomena. For example, the reaction between $\mathrm{H}_{2}$ and $\mathrm{Cl}_{2}$ on condition of illumination is instant, so it's difficult for students to catch the process and phenomenon, while some other experiment reaction is so slow that the phenomenon is not obvious, such as polysaccharide hydrolysis and esters hydrolysis. Therefore, students always fail to get the expected effect leading to students' low passion for chemistry learning. In order to overcome the disadvantage, we can slow down experiments that react fast on the big screen by video projector, so that students can observe details of experiment process, oppositely, the slow reaction experiment can be adjusted and controlled to make the reaction fast and enhance the visibility.

\section{Showing Micro Experiment}

Chemistry is a branch of science researching on constitutes property and regularity of substance in nature. Therefore, the subjects of chemistry research are mainly about microstructures of materials, the motion and change of particles, reaction principle and process of chemistry. We cannot see the microstructure and micro-particle with our naked eyes. The essence of chemical change is the recombination of primary atoms by chemical bonds formation and break. These contents are so abstract that it's hard for students to understand. To solve the problem, we can turn to multimedia courseware made by Multimedia animation and three-dimensional pictures for help, by which the microstructures, micro-particles and processes of chemical changes can be vividly demonstrated. The multimedia technology can offset the shortage that chemistry experiment cannot show the dynamic change in the microscopic world. For example, the three-dimension animation can be applied to simulate the structure and process of bond formation. What's more, different atoms can be represented by different color balls, and the scale of the ball represents the outermost electron. So the formation of ionic compounds and covalent compound can be simulated clearly. For another example, the three-dimension animation and pictures can be applied to show the rotation, motion and scale of crystal space structure and its stretch. Moreover, the direction of election current and cationanionic in primary battery can be magnified by the three-dimension animation and pictures.

Analyzing the formation and break of bond in the molecular structure is the key point in organic reaction process. Application of multimedia technology in organic reaction process will make its effect much better. The formation and break process of chemical bonds can be shown visually and clearly by multimedia animation. For instance, the peptide formation process of amino acids can be designed into animation form. When two amino acids meet the covalent bond between carbon and hydroxyl group breaks into two free groups in one amino acid, at the same time, the covalent bond between nitrogen and hydrogen breaks into two free groups in the other amino acid, and then the four free groups are rearranged to form a dipeptide and a water molecule.

\section{Showing Toxic and Pollutional Experiments, Making Experiments Clean}

There are some toxic substances involved in some chemistry experiments, such as potassium cyanide, sodium nitrite, benzene and carbon monoxide. There are also some toxic gas produced in some experiments, such as cyanic acid and sulfur dioxide. There may be some waste water, exhaust fumes and solid waste in the process of experiment. This kind of experiment can produce large volumes of gas, leading to environment pollution and harming the health of teachers and students. It's a good way that students can do the experiment by computer. In this way, students can master experimental method easily and the experiments are also cleaner. Meanwhile, it can enhance students' environmental awareness and set up concept of green chemistry.

\section{Simulating Wrong Operations, Showing Inflammable and Explosive Experiments to Make Experiment Safe}

There's no doubt that it's dangerous for students to perform inflammable and explosive experiments in the lab. Meanwhile, demonstrating such dangerous experiments is also not suitable. The multimedia technology can deal with this problem. The inflammable and explosive experiment can be done with the help of the application of multimedia technology, which keeps students and teachers safe. The wrong operation can be shown on computer by video technology. For example, students can simulate adding water to dilute concentrated sulfuric acid during the experiment of dilution of sulfuric acid. As a result, the sulfuric acid solution splashes around. As another example, after finishing the process of producing hydrogen experiment with the device of gas generator explodes as soon as students ignite the new produced hydrogen without testing its purity. Student can experience dangers that wrong operation brings, which enhances the awareness of danger and help student master the correct steps of experiment, and more attention will be paid to wrong operation in the experiment.

\section{Materialization and Visualization of Abstract Knowledge}

It's good to review theoretical knowledge related to experiment before entering the lab. This helps students understand principles, purposes and methods of an experiment. But it's hard for students to understand them when teachers just narrate the theoretical knowledge (Wang \& Liu, 2010). Students can finish the experiment successfully based on solid theoretical knowledge which can be shown by projector, such as molecular structure, orbital shapes and crystal structure. To some extent, three-dimensional figure is much better than chemistry model for solving these chemistry problems. 
Chemistry model can be made by some map-making software, such as AutoCAD, flash, 3Dmax, ChemDraw and Chem3D. For example, when illustrating production principle of chlorine in class, The method of combining overall cognition and part analysis was applied in the teaching process, with the help of simulation experiment. First, a series of questions arise from presenting pictures of sea, such as what sea water tastes like, what is contained in the vast sea, and what the edible salt eaten in daily life is made from. Second, the industrial method of making chlorine is introduced to the students by explaining from electrolysis of water to the electrolysis of saturated salt solution. Finally, the chlorine preparation experiment is simulated by the Jinhuake simulation chemistry software. In this process, students' mind was inspired by Q\&A. On one hand, from the point of our daily life, it prevents poisonous gas from polluting the Earth. On the other hand, simulation experiment is a good way for improving economic performance, especially for cost-saving chemicals. Stick model of methylbenzene can be made by software of Jinhuake simulation chemistry lab (as shown in Figure 1). How to get started using Jinhuake simulation chemistry is important to teachers when making molecular models, Jinhuake simulation chemistry, made in China, is a kind of software for middle school chemistry instruction. The software includes three modules of simulation chemistry lab, three-dimensional molecular model and chemistry encyclopedia. The steps of making stick model of methylbenzene are as follows:

After the three modules of simulation chemistry lab are re- moted, the first step is to choose the benzenoid the red arrow points to from the toolbar on the left. The ball-and-stick model of benzene is shown on the interface (as shown in Figure 2). In order to get methylbenzene, it needs to choose one of the hydrogen atoms of benzene to substitute methyl group for hydrogen atom, and the hydrogen atom turns white (as shown in Figure 3). After that, the ball-and-stick model of methylbenzene is shown on the interface (as shown in Figure 4). In addition, the three-dimensional space distribution of ball-and-stick model of methylbenzene can be shown by rotating it, one of the shape of ball-and-stick model of methylbenzene is shown on the interface (as shown in Figure 5). What's more, it's convenient to view the animation of its three-dimensional space by recording animation and the shape of ball-and-stick model of methylbenzene can be saved easily (as shown in Figure 6).

The three-dimensional animation of sp3 hybrid orbit can be shown vividly. It can be rotated optionally. When relating to structure isomeride in class, teachers can assemble and disassemble the molecular structure, and the scale of molecular structure can be changed with the application of map-making software (Liu, Jiang, \& Xu, 2003). Moreover, the model made by map-making software can be reapplied in class instead of actual model again and again, which can save costs (Wang \& Zeng, 2006). The invisible substance in process of experiment can be visible in simulation, and some experiments that cannot be observed directly have become true. This kind of software makes it come true while other media cannot (Chen, 2008).

Schools place emphasis on developing students' comprehensive

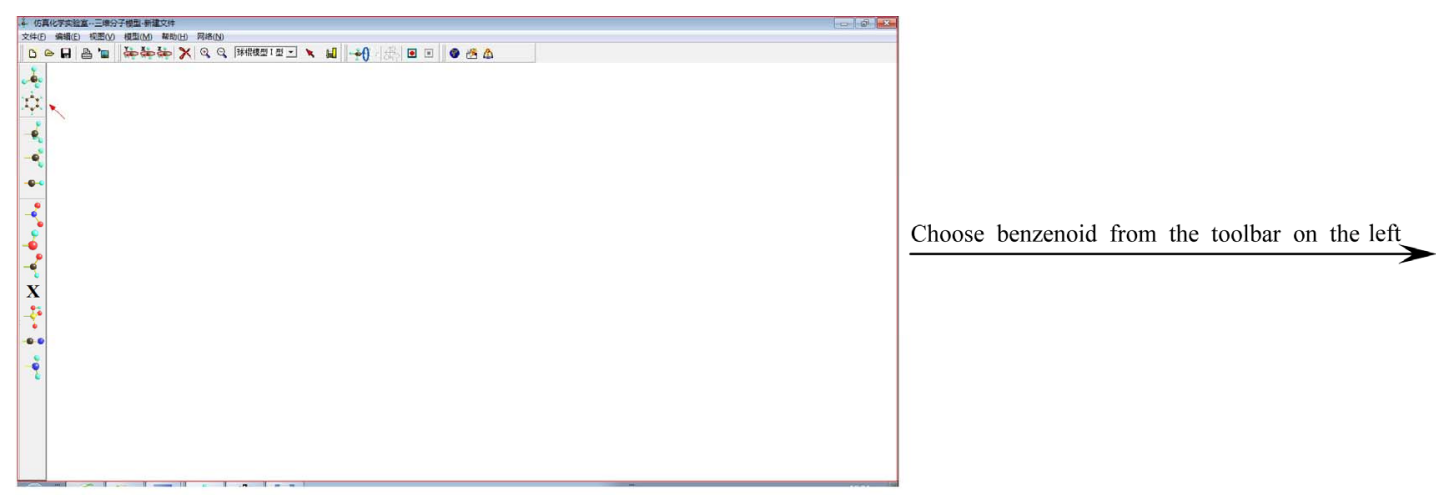

Figure 1.

Interface of Jinhuake simulation chemistry lab.

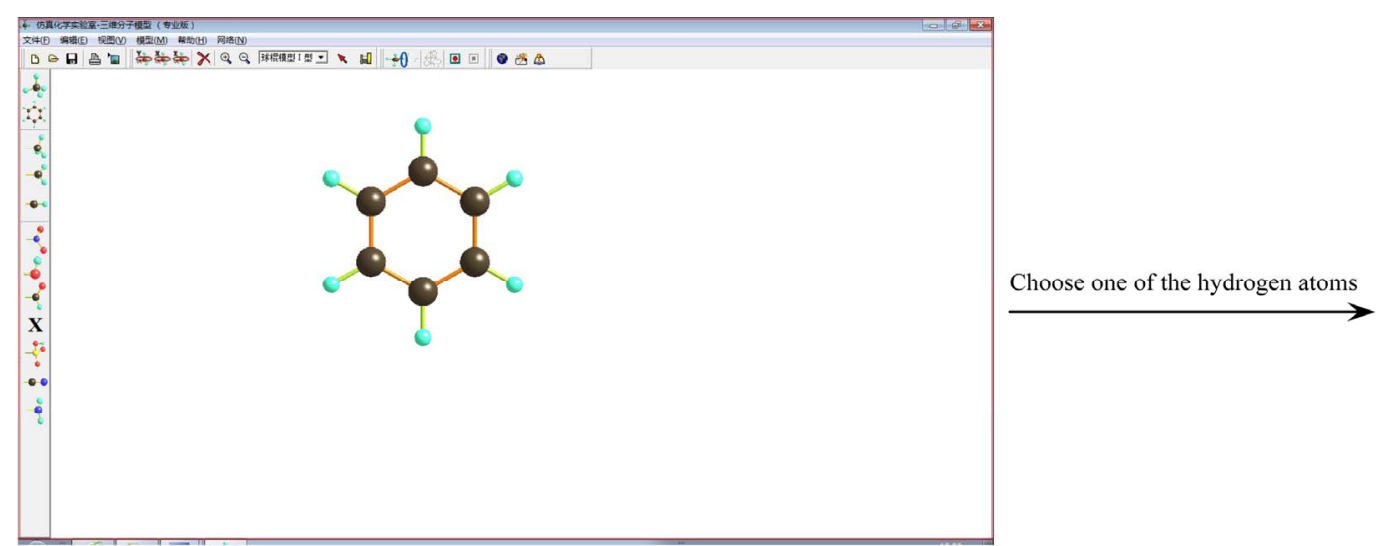

Figure 2.

Ball-and-stick model of benzene. 


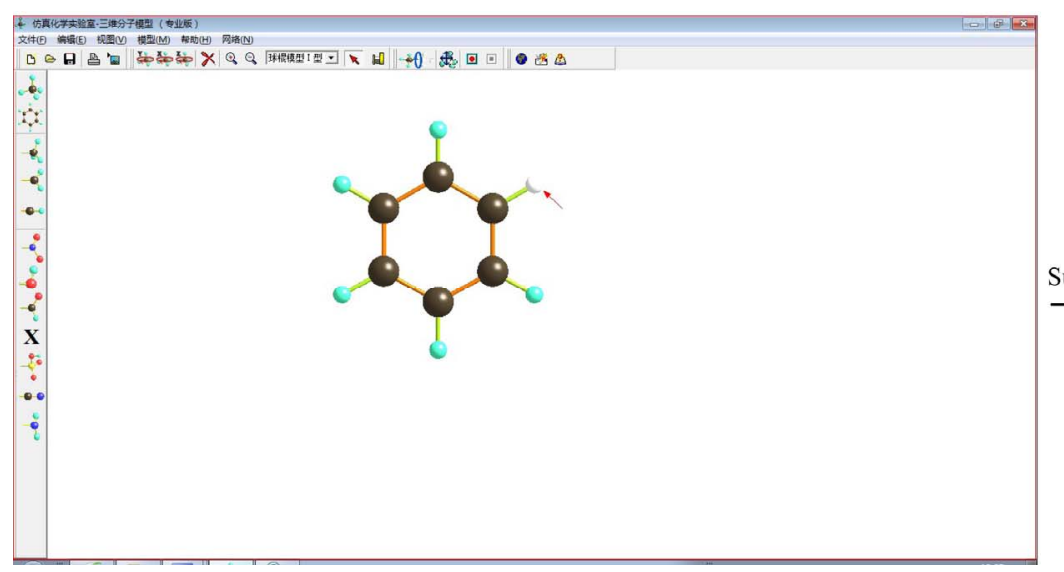

Figure 3.

Substitud methyl group for hydrogen.

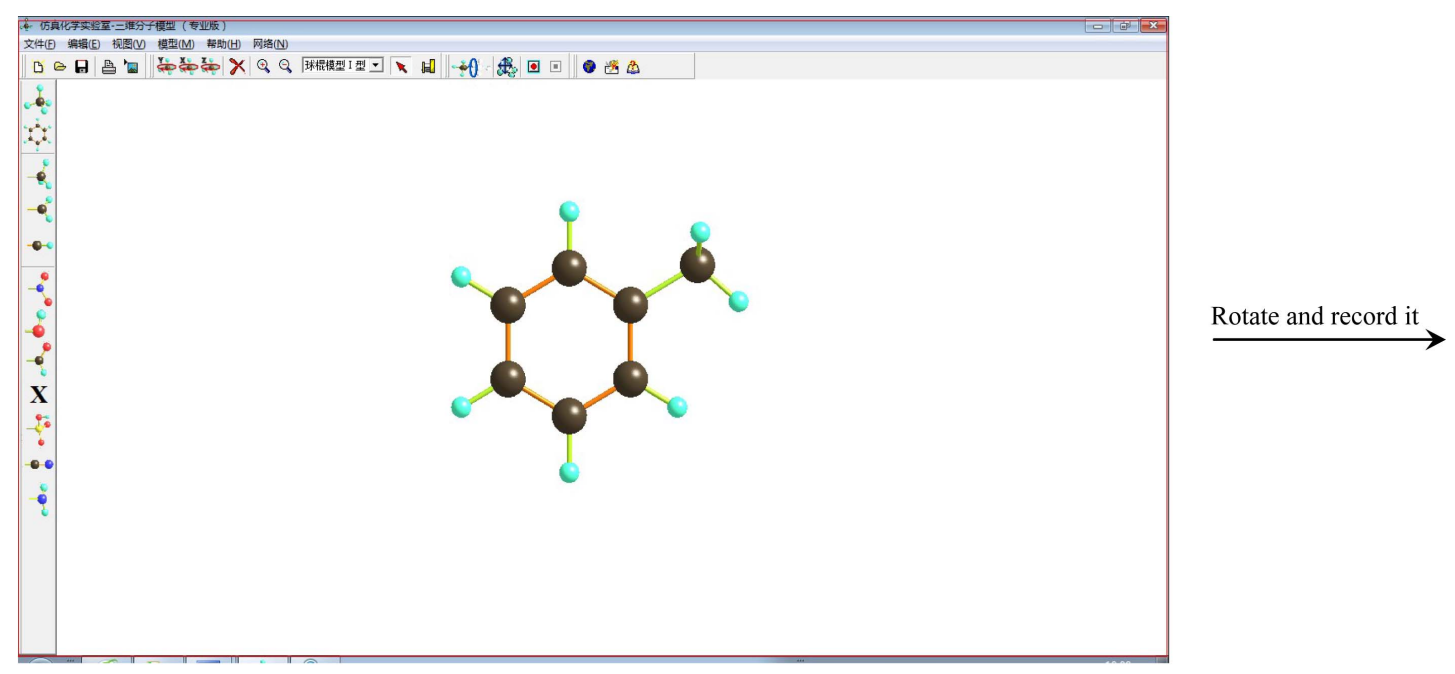

Figure 4.

Ball-and-stick model of methylbenzene.

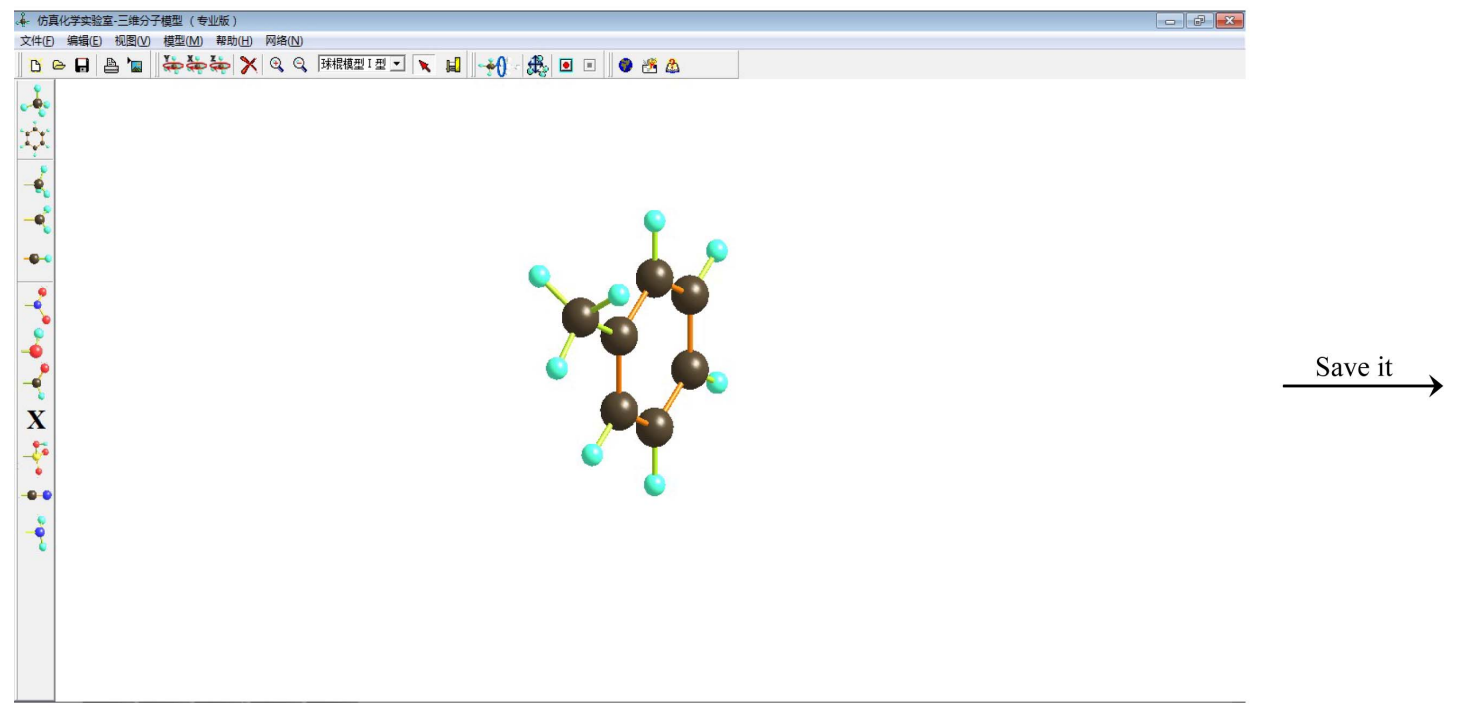

Figure 5.

Another shape of methylbenzene. 


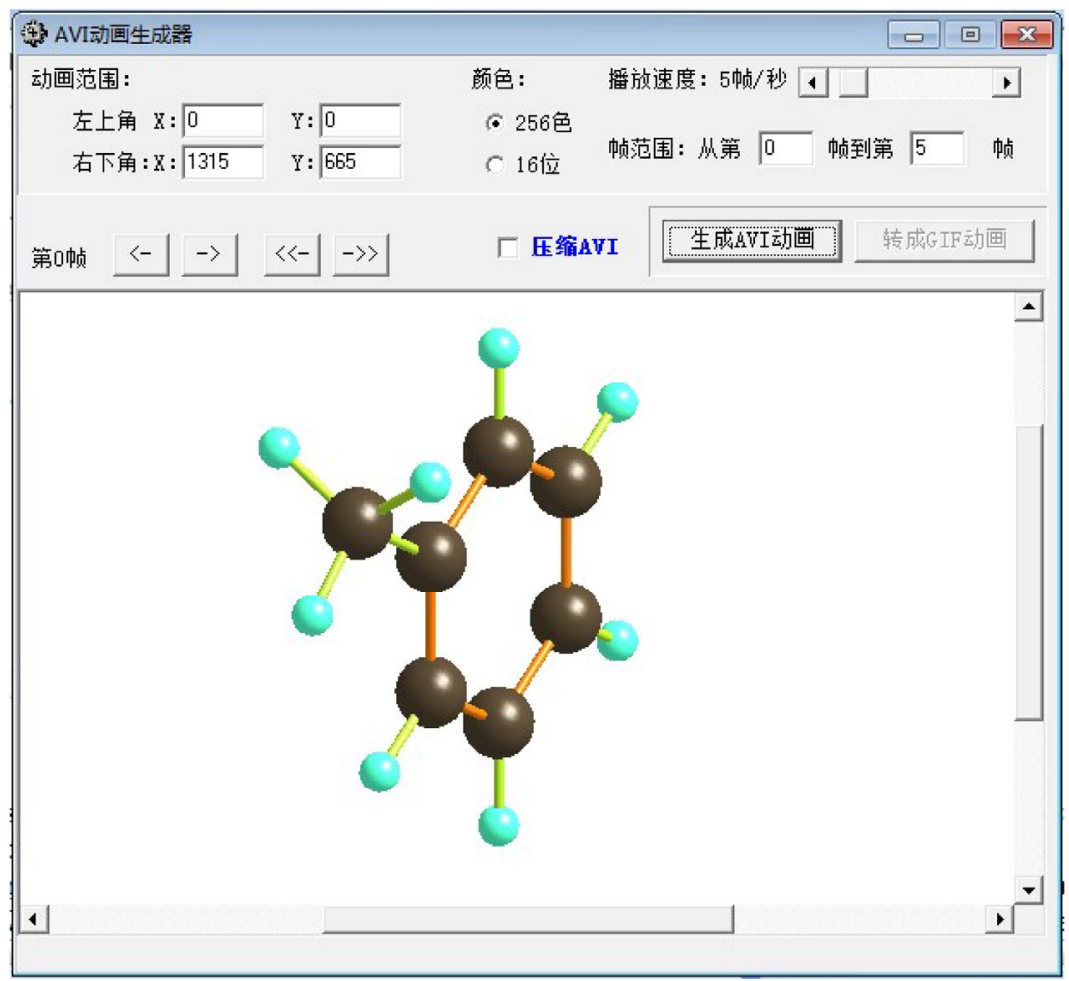

Figure 6.

The interface of saving.

experiment ability by reforming chemistry experiment. The number of exploring experiment has increased while the number of verification experiment has been cut down. In order to make up for the shortage of verification experiment, some important verification experiment can be recorded by video camera, such as properties experiment of ethylalcohol and phenol (Wang, 2006). The advantage of video camera is that it can help students understand the properties of substance completely. According to teaching and research, it achieved good teaching results.

\section{Showing Instrumental Analysis Experiment, Alleviating Instrument Shortage}

Lacking instrument in instrumental analysis experiment has been a big problem in the middle schools of China, especially in rural schools. The speed of upgrading products is quite low. Class size is large in China. Multimedia teaching software can be applied to instrumental analysis experiment. Students can master elementary principles and operating procedure by means of simulating instrumental analysis experiment on computer (Zhang, Zhang, \& Luo, 2003). Familiar with the instrument, students can accomplish experiments quickly. It's also good for the maintenance of instrument.

\section{Showing Large Scale Experiment and Technological Process}

Constrained by conditions, time and space, it's hard to show large-scale and complicated technological process, such as industrial production of sulfuric acid, ammonia and synthetic rubber. We can simulate the process by multimedia technology.
The internal structures of equipments, the liquid status and the principle of experiments can be imitated vividly by two-dimensional and three-dimensional animation. Students can master the operation method vicariously and know how to deal with accidents often occurred in practice.

\section{Operation Skills Practice and Reinforcing the Regularity of Students' Basic Operation}

As we all know, basic operations is one of the most important experimental contents. Students get the skills of basic operations in class with the help of teachers demonstrating the operation in details. However, not everyone can clearly grasp it, especially for those difficult and complex experiments, because of the large number of students and their different positions in class. It takes much time and effort for teachers to demonstrate these experiments satisfactorily. In order to avoid these problems, teachers can show the basic operations with video technology, such as clearing and assembling laboratory glassware and weighing with analytical balance.

\section{Assisting Experiment Design, Developing Students' Innovation Ability}

Doing experiment has a positive influence on students' scientific cultivation and qualities. Students can design experiments by themselves in simulation laboratory. Different experiment schemes can be designed to solve problems with the help of computer multimedia technology, and updated when errors occur. There are many experiment equipments worthy of being designed, such as designing equipment of gas production, impurity removing gas and drying gas. The multimedia tech- 
nology is available when funds limit materials to conduct live experiments. This is a problem that exists in many regions of the world, including schools in the US. The wrong operation can also be corrected by suggestion to improve students' ability of experimental design. Students can repeat experiments many times without considering the limitation of instrument and chemicals.

\section{Issues about the Application of Multimedia Technology in Chemistry Experimental Instruction}

\section{Real Experiments Cannot Be Replaced}

Chemistry is an experimental science. It arouses the students' study interests and cultivates their practical abilities. Simulation laboratory is economical, simple and safe. However, it only makes students know rather than do experiments. Simulation laboratory is a kind of ideal model in which students cannot experience failure, and there is little chance to think and analyze causes of failure. Therefore, we should consider simulation laboratory an extension and supplement to real experiments.

\section{Properly Dealing with the Relationship among Teachers, Students and Multimedia}

The traditional chemistry teaching is a kind of teaching mode which takes books as the carrier, and teachers as the center, where teachers' interpretation and students' attending the lecture and practice are the main activities. Teachers play an important role as organizer, helper and promoter. Students are active constructors of knowledge in classes. The task of multimedia is creating study environment and arousing students' study motivation. It is a cognitive tool for students to actively learn and explore (Zhao \& Zhu, 2008). Multimedia technology should be used reasonably in accord with students' cognitive ability. And it can inspire the students' studying interest and innovation consciousness when properly designed. If the position and function of all elements of teaching can be located, we will make good use of multimedia.

\section{Making Multimedia Courseware Scientific}

Whether the courseware is scientific or not is an important parameter for the courseware evaluation. Scenarios simulation not only creates a lively atmosphere but also promotes the development of teaching from static state description to dynamic description (Zhao \& Yu, 2006). Simulation laboratory is used based on experimental facts, but it cannot take the place of experimental practice (Ren, 2002). The purpose of courseware is to impart knowledge, which can be mastered well by students. So it's quite necessary to make sure that the content is correct. There is no mistake in the respect of knowledge, or it brings negative effects on teaching.

\section{Prospective Forecast of Multimedia Technology in Chemistry Experimental Instruction in China}

\section{Developing towards Intelligent Direction}

Artificial intelligence is developing rapidly and it can be applied widely to education. A computer acts as a teacher by interactive question-and-answer in a new environment of learning.
Students are encouraged ask questions as many as possible. What's more, artificial intelligence can judge the results of a test and correct it instantly. According to students' learning style, the computer can adjust teaching strategies to control the teaching process to meet students' learning demand. However, it's really rare for artificial intelligent technology to apply in chemistry instruction in China. It is still in development there is no doubt that it will be a hot spot in the field of multimedia technology soon (Hu, 2002).

\section{Developing towards E-Learning System}

Students are regarded as active constructors of knowledge in e-learning system, it's free for them to choose contents and gain abundant study materials and convenient to consult teachers online. Tele-collaborative learning comes true finally. Computer Assisted Instruction (for short CAI) will be one of the most important developing directions, especially for the webbased research studying.

\section{Applying Virtual Reality Technology to Teaching}

At present, virtual physical laboratory and virtual chemistry laboratory have been developed in foreign countries. Virtual reality is an interactive artificial environment. Users communicate with each other by visions, sounds and touch on computer. And they feel and experience what they're going through with helmet, data gloves, data glasses, and three-dimensional mouse in such an interactive artificial environment. By operating experiments, users acquire the knowledge and skills and achieve goals beyond traditional teaching. Virtual reality will be applied in the middle school class in China in the near future.

\section{Conclusion}

As a modern teaching technology, multimedia techniques can bridge the gap of traditional chemistry experiment teaching model. The application of multimedia techniques in chemistry experiment has not only changed traditional teaching mode, but also improved teaching efficiency. Once Multimedia Technology is used in "traditional chemistry teaching", the teaching will show advantages of Multimedia Technology teaching as well as traditional teaching. Multimedia technology can push the innovation of school teaching and can develop towards the direction of science and modernization. It becomes more acceptable and offers a solution for shrinking equipment budget, which is a challenge that many schools are facing. However, the importance of traditional chemistry teaching can not be ignored. It should be emphasized that computer-assisted instruction cannot take the place of real experiment though it is a good method in teaching. The application of multimedia technology in chemistry experimental instruction is unreasonable and defective in China. However, computer assisted instruction of multimedia technology in China has a bright future for development. It's an important subject for further research on how to develop and improve computer assisted instruction of multimedia technology.

\section{REFERENCES}

Chen, X. (2008). The application of computer assisted instruction in chemistry teaching. Exam Week, 19, 166-167.

He, X. P. (2007). Application of computer assisted instruction in high 


\section{M. CHEN ET AL.}

school chemistry teaching. Reading and Writing, 8, 122-123.

$\mathrm{Hu}, \mathrm{X}$. (2002). Application and prospect of computer assisted instruction. Education in Chemistry, 12, 24-25.

Huang, X. Y. (2007). The application and thoughts of multimedia technology in the teaching of experiment chemistry. China Modern Educational Equipment, 6, 135-138.

Liu, J. F., Jiang, Y. CH., \& Xu, L. F. (2003). The application of computer assisted instruction in chemical experimental lessons. Journal of Liaoning Agricultural Vocation-technical College, 6, 52-53.

Ma, W. L., \& Ran, M. (2008). The application of a new chemistry courseware in scientific research. Chemistry Education, 1, 58-59.

Wang, H. M., \& Zeng, X. H. (2006). The application of computer assisted instruction in chemical experimental teaching. Equipment and Experiment in Primary and Secondary, 5, 2-3.

Wang, J., \& Liu, Q. Y. (2010). Preliminary discussion on the function of computer assisted instruction in chemical experiment classes. Journal of Shangrao Normal University, 3, 62-65.

Wang, S. CH. (2008). Application of computer-aided instruction in teaching. Exam Week, 50, 200-201.

Wang, Z. H. (2006). Experimental chemistry (pp. 82-85). Nanjing: Jiangsu Educational Press.

Yan, X. Q., Yu, Q. S., \& Sun, B. (2005). Application of CAD method to experimental instruction of physical chemistry. Research and $E X$ ploration in Laboratory, 4, 36.

Zhang, D., Zhang, J. H., \& Luo, C. J. (2003). Designing and making multiple-media teaching software of instrumental analysis. Experimental Technology and Management, 4, 66-68.

Zhao, F. Q., \& Zhu, Y. H. (2008). Application of multimedia courseware in chemistry teaching. China Science and Technology Information, 3, 236-239.

Zhao, F. Z., \& Yu, L. L. (2006). Designing and implementing of oil field security operation simulation system based on virtual reality technology. Computer Systems Applications, 11, 6-9.

Ren, D. F. (2002). The development and status of CIA study. Journal of Sichuan Three-Gorges University, 5, 104-107. 